\title{
On the correlation of seismic activity to syzygies.
}

\author{
Mikhail Kovalyov ${ }^{1}$ \\ Received 11 October 2018; accepted 12 November 2018; published 14 February 2019.
}

There must be hundreds of papers dedicated to the studies of the relationship between lunar phases and seismic activity on Earth; half of them claim to prove that lunar phases affect seismic activity on Earth, while the other half disprove the same. It is shown here that the lunar phases indeed affect seismic activity on Earth, the correlation between them is especially easily seen in magnitude $\geqslant 8.2$ earthquakes and VEI $\geqslant 5$ volcanic eruption, but only seasonally. The seasons of correlation last centuries and are intimately connected to the behavior of the Earth's magnetic field. The correlation between the lunar phases and seismic activity may be also easily observed in the seismic activity away from well-formed tectonic lines, e. g. the earthquakes along the East African Rift and high-magnitude deep earthquakes. KEYWORDS: earthquakes; volcanic eruptions; syzygies.

Citation: Kovalyov, Mikhail (2019), On the correlation of seismic activity to syzygies., Russ. J. Earth. Sci., 19, ES1005, doi:10.2205/2019ES000650.

\section{Introduction}

A simple online search for the correlation of powerful seismic events with syzygies yields literally an enormous number of research papers going back to, at least, the 19th century, [Klotz, 1914; some prove the existence of such a relationship while the others disprove it.

Rejections of the very existence of such correlation are plentiful both in the scientific literature and online, with any correlations attributed to mere coincidence. The existence of correlation between seismic activity and lunar motion is a seismic stand-off between those who believe that seismic activity can be predicted and those who do not. The former are best represented by the International Institute of Earthquake Prediction Theory founded by Vladimir Keilis-Borok, whose algorithm for earthquake prediction has been used to successfully predict the outcomes of numerous

\footnotetext{
${ }^{1}$ Professor Emeritus, University of Alberta, Canada

Copyright 2019 by the Geophysical Center RAS. http://rjes.wdcb.ru/doi/2019ES000650-res.html
}

elections. The latter are represented by western institutions, who, after remarkably unsuccessful attempts to predict earthquakes in 1970s, have switched to a complete denial of any seismic predictions. The latter argue that the increase in the tidal pull near syzygies is too small to have any effect. However, quite a few physical phenomena exhibit drastic responses to rather tiny changes in parameters. One example is provided by $\mathrm{CO}_{2}$ which can be liquefied at $\approx 304.25 \mathrm{~K}$ at a pressure of $\approx 73 \mathrm{~atm}$, but not at a slightly higher temperature, even under pressures as high as $3000 \mathrm{~atm}$; of course, that is because $\approx 304.25 \mathrm{~K}, \approx 72.9$ atm is $\mathrm{CO}_{2}$ 's critical point. If the Earth is in a critical state in some sense, it may be sensitive to relatively small changes in the forces exhorted on it.

Can we detect the effect of syzygies? Our research shows that it is indeed possible to detect tidal influence on Earth's seismic activity, yet it is often obscured by other factors. In this paper we discuss the seismic activity showing correlation with lunar syzygies, specifically 1 ) magnitude $\geqslant 8.2$ earthquakes, 2) VEI $\geqslant 5$ volcanic eruptions, 3) earthquakes in Africa, 4) earthquakes originating below $400 \mathrm{~km}, 5$ ) the year 2012 phenomenon. 


\section{Data Sources Used}

The seismic data seems to be reliable from 1978 onwards; the first disagreements between major catalogs of seismic data appear as recently as 1977, e. g. USGS and NOAA catalogs (Table 1) disagree on the magnitude of the 1977/8/19 earthquake, the earthquake is given magnitude 8.3 by USGS and magnitude 8.0 by NOAA. With a considerable leap of faith we may assume that the seismic data from 1900 onwards is sufficiently reliable to draw conclusions. The pre-1900 seismic data cannot be considered reliable and may be used to draw only the most general conclusions.

Author has compiled a data base of seismic activity versus lunar phases from open sources; the data is arranged in ten tables [Kovalyov, 2019 published in Earth Science Data Base, created and managed by GCRAS.

The sources of data for each table are provided in the note to the table.

\section{Data Analysis}

The main ingredients of the patterns associated with the lunar motion are the synodic and anomalistic months. A synodic month is the time between two exactly the same adjacent phases of the Moon, e.g. two adjacent Full Moons, two adjacent New Moons, etc.; an anomalistic month is the time between two adjacent perigees. The exact values of synodic and anomalistic months vary, but the average synodic month is $\approx 29.530587981$ days and the average anomalistic month is $\approx 27.554551$ days. Since 14 average synodic months $\approx 413.428$ days and 15 average anomalistic months $\approx 413.318$ days are almost the same, the lunar motion is almost cyclicly repeating itself approximately every 413414 days. Thus we may define a full lunar cycle to be a period of $\approx 413$ consecutive days which begins and ends with the same lunar phase and contains 14 New Moons, 14 Full Moons, and 15 perigees.

The closest perigee and 2nd closest perigee of a full lunar cycle come within less than 11 hours of New/Full Moon, and are typically separated from each other by 6-8 synodic months, (https://www. fourmilab.ch/earthview/pacalc.html e.g. the closest perigee and Full Moon were less than 2 hours apart on $2015 / 9 / 28$, the 2 nd closest perigee and
New Moon were less than 8 hours apart on $2015 / 2 / 19$. Less often, the 2 nd closest perigee may be merely a month away from the closest perigee, e. g. in 1963 the perigees on $1963 / 11 / 2$ and $1963 / 11 / 30$ were at, correspondingly, $356958 \mathrm{~km}$ and $356954 \mathrm{~km}$ with, correspondingly 10 hours 30 minutes and 10 hours 47 minutes separating the perigees from Full Moon; the third closest perigee of $356972 \mathrm{~km}$ was on 1963/4/23 with only two hours away from New Moon. Seismic activity is influenced not only by mere proximity to a syzygy, but also by different aspects of the syzygy, e. g. the distance between Moon and Earth, proximity to perigee/apogee, proximity to perihelion, etc. In this paper, for simplicity's sake, we divide syzygies into only three types: 1) regular syzygy is a syzygy more than 12 hours away from the nearest perigee; 2) syzygy-closest/2nd closest perigee is a syzygy within 11 hours of a closest/2nd closest perigee; 3) twin pair is a syzygy within 11 hours of a closest/2nd closest perigee coupled with an adjacent syzygy with very similar parameters, e.g. $1963 / 11 / 2$ and $1963 / 11 / 30$ and define $\mathcal{H}_{\nu}$ to be the time either within $30+\nu$ days of a syzygyclosest/2nd closest perigee, or within $30+\nu$ days of a twin pair, here 1 day $=24$ hours.

An example of a twin pair is provided by the already mentioned 1963/11/30 Full Moon separated from the closest perigee of $356954 \mathrm{~km}$ by 10 hours 47 minutes and 1963/11/2 Full Moon separated from the 2nd closest perigee of 356958 $\mathrm{km}$ by 10 hours 30 minutes; they were preceded by the $1963 / 4 / 23$ New Moon separated from the 3rd-closest perigee of $356972 \mathrm{~km}$ by merely two hours. For our purpose, the three events are practically indistinguishable, all three should be treated as syzygy-closest/2nd closest perigees; it is easier, however, to view the two syzygy-perigees of $1963 / 11 / 30$ and $1963 / 11 / 2$ as a single event of a twin pair.

We say that an event is in $\mathcal{H}_{\nu}$, if it is either within $0.5+\nu$ days of a regular syzygy or within $30+$ $\nu$ days of a syzygy-closest/2nd closestperigee or a twin pair;; $\mathcal{H}_{\nu} \subset \mathcal{H}_{\nu+1}$. To estimate the percentage of days in $\mathcal{H}_{\nu}$, consider the period of 1004 average synodic months $\approx 1004 \times 29.530587981 \approx 81$ years 63.46033 days or 1076 average anomalistic months $\approx 1076 \times 27.554551 \approx 81$ years 63.446488 days with a year taken to be 365.25 days. The difference between 81 years 63.46033 days and 81 years 63.44688 days is $\approx 0.01345$ days or slightly more 
than half an hour so for any practical purposes we may consider the two periods to be equal to 81 years 63.5 days or 29648.7 days and contain 2008 syzygies. The period will contain $\frac{1004}{14} \times 2=$ 144 full lunar cycles, almost each cycle has two syzygy-closest/2nd closest perigees, but some cycles may have a syzygy-closest/2nd closest perigee and a twin pair of syzygies which consists of two syzygy-perigees separated by a synodic month, e.g. $2002 / 2 / 27$ and $2002 / 3 / 28$. As a sample of such a period we may take $1921 / 1 / 1-2002 / 2 / 4$, it contains 2008 syzygies comprising 1851 regular syzygies, 133 syzygy-closest/2nd closest perigees, 12 twin pairs of syzygy-perigees. The number of days of $1921 / 1 / 1-2002 / 2 / 4$ in $\mathcal{H}_{\nu}$ is given by the formula $1851(0.5+2 \nu)+133(60+2 \nu)+12(89.5+$ $2 \nu)=9979.5+3992 \nu$. Thus the days in $\mathcal{H}_{\nu}$ make $9979.5+3992 \nu$

29648.7 portion of $1921 / 1 / 1-2002 / 2 / 4$; we take it as a reference formula for all periods of time:

$$
\begin{gathered}
\approx \frac{9979.5+3992 \nu}{29648.7} \approx\left\{\begin{array}{l}
74.1 \%, \text { if } \nu=3 \\
60.6 \%, \text { if } \nu=2 \\
47.1 \%, \text { if } \nu=1 \\
33.7 \%, \text { if } \nu=0
\end{array}\right. \\
=74.1 \% / 60.6 \% / 47.1 \% / 33.7 \%
\end{gathered}
$$

If a sufficiently large group of events has $p_{3} \% / p_{2} \% /$ $p_{1} \% / p_{0} \%$ in $\mathcal{H}_{3} / \mathcal{H}_{2} / \mathcal{H}_{1} / \mathcal{H}_{0}$, then the ratios $\boldsymbol{\kappa}_{3}=$ $\frac{p_{3}}{74.1}, \boldsymbol{\kappa}_{2}=\frac{p_{2}}{60.6}, \boldsymbol{\kappa}_{1}=\frac{p_{1}}{47.1}, \boldsymbol{\kappa}_{0}=\frac{p_{0}}{33.7}$ are indicative of the syzygies' influence on the events.

For the sake of brevity, we shall use M for "magnitude".

\section{$M \geqslant 8.2$ earthquakes in $1550-2017$}

Table 1 shows $M \geqslant 8.2$ earthquakes in 1938 2017. It was compiled by merging data from USGS and NOAA; fore/aftershocks are not listed.

Of the 36 earthquakes in Table 1 listed by USGS as $\mathrm{M} \geqslant 8.2,34 / 29 / 23 / 18$, or $94.4 \% / 80.6 \% / 63.9 \% /$ $50 \%$, are in $\mathcal{H}_{3} / \mathcal{H}_{2} / \mathcal{H}_{1} / \mathcal{H}_{0}$; the ratios of these percentages to $(1) \kappa_{3}=\frac{94.4}{74.1} \approx 1.27, \kappa_{2}=\frac{80.6}{60.6} \approx$ $1.33, \kappa_{1}=\frac{63.9}{47.1} \approx 1.36, \kappa_{0}=\frac{50 .}{33.7} \approx 1.48 \mathrm{in}-$ crease as $\nu$ decreases. Only two earthquakes from
USGS did not make it to $\mathcal{H}_{3}$, both rather special. The 2006/11/15 earthquake, followed by an $\mathrm{M}=8.12007 / 1 / 13$ aftershock, started the elevenmonth season $2006 / 11 / 15-2007 / 9 / 29$ with the most $M \geqslant 8.0$ earthquakes, five in total; for comparison there were $69 \mathrm{M} \geqslant 8.0$ earthquakes in 1938-2017 averaging $\approx 0.79$ earthquakes per an eleven-month period. The 1977/8/19 earthquake started the $1977 / 8 / 20-1985 / 3 / 3 \mathrm{M} \geqslant 8.0$ earthquake drought, the longest period in 1900-2017 without a $M \geqslant 8.0$ earthquake. Of the 39 earthquakes in Table 1 listed by NOAA as $\mathrm{M} \geqslant 8.2,38 / 33 / 27 / 20$, or $97.4 \% / 84.6 \% / 69.2 \% / 51.3 \%$, are in $\mathcal{H}_{3} / \mathcal{H}_{2} / \mathcal{H}_{1} / \mathcal{H}_{0}$; the ratios of these percentages to (1) $\kappa_{3}=\frac{94.4}{74.1} \approx$ $1.27, \kappa_{2}=\frac{84.6}{60.6} \approx 1.4, \kappa_{1}=\frac{69.2}{47.1} \approx 1.47, \kappa_{0}=$ $\frac{51.3}{33.7} \approx 1.52$ increase as $\nu$ decreases. Since the $1977 / 8 / 19$ earthquake is listed by NOAA as having $M<8.2$, only the $2006 / 11 / 15$ earthquake is left out of $\mathcal{H}_{3}$. The 2006/11/15 earthquake preceded the 2006/12/5 X9.0 solar flare, [Kovalyov, 2019, Table 1]; whether that is a pure coincidence or there is a connection between the earthquake and solar flare is not clear. In 1957-1965 $\mathrm{M} \geqslant 8.2$ earthquakes struck practically every year, all but one in $\mathcal{H}_{0}$; the years coincided with the 19 th primary solar cycle of 1955-1965, the most powerful primary solar cycle since the record-keeping began in 1700.

Table 2 shows $\mathrm{M} \geqslant 8.2$ earthquakes in 1900 1933. It was compiled exactly the same way as Table 1. Of the 12 earthquakes from USGS $5 / 3 / 2 / 2$, or $41.7 \% / 25 \% / 16.7 \% / 16.7 \%$, are in $\mathcal{H}_{3} / \mathcal{H}_{2} / \mathcal{H}_{1} / \mathcal{H}_{0}$; the ratios of these percentages to (1) $\kappa_{3}=\frac{41.7}{74.1} \approx$ $0.56, \boldsymbol{\kappa}_{2}=\frac{25}{60.6} \approx 0.41, \boldsymbol{\kappa}_{1}=\frac{16.7}{47.1} \approx 0.35, \boldsymbol{\kappa}_{0}=$ $\frac{16.7}{33.7} \approx 0.5 ;$ the portion outside of $\mathcal{H}_{3}$ got $\frac{1-41.7}{1-74.1}$ $\approx 2.25$ times its fair share of earthquakes. Of the 27 earthquakes from NOAA, 15/12/8/6, or 55.6\%/ $44.4 \% / 29.6 \% / 22.2 \%$, are in $\mathcal{H}_{3} / \mathcal{H}_{2} / \mathcal{H}_{1} / \mathcal{H}_{0}$, the ratios of these percentages to (1) $\kappa_{3}=\frac{55.6}{74.1} \approx$ $0.75, \kappa_{2}=\frac{44.4}{60.6} \approx 0.73, \kappa_{1}=\frac{29.6}{47.1} \approx 0.63, \kappa_{0}=$ $\frac{22.2}{33.7} \approx 0.66 ;$ the portion outside of $\mathcal{H}_{3}$ got $\frac{1-55.6}{1-74.1} \approx 1.71$ times its fair share of earthquakes. Instead of being attracted to syzygies, the earth- 
quakes were repelled from syzygies; we may call such behavior anti-correlation. Both catalogs lead to $\boldsymbol{\kappa}_{3} \geqslant \boldsymbol{\kappa}_{2} \geqslant \boldsymbol{\kappa}_{1} \leqslant \boldsymbol{\kappa}_{0}$. The thirteen earthquakes in Table 2 that did not make it to $\mathcal{H}_{3}$ may be divided in clusters: three in $1901 / 8 / 9-1903 / 6 / 2$; three in $1905 / 7 / 9-1906 / 1 / 31$; three in $1917 / 5 / 1$ 1918/8/15; and two in 1922/11/11-1924/4/14.

There is no reliable data about the pre-1900 earthquakes, the magnitude, coordinates of the epicenter, or even date of pre-1900 earthquakes often vary widely between catalogs. The reason for that is that the pre-1900 earthquake data is compiled based on historical descriptions, often very vague and subjective; the interpretations of these descriptions are also very subjective. The first glance at pre- $1900 \mathrm{M} \geqslant 8.2$ earthquakes is provided by Table 3 comprising the most-gossipedabout $1550-1899 \mathrm{M} \geqslant 8.2$ earthquakes compiled from two articles in Wikipedia; the sheer number of non-scientists involved in Wikipedia seems to guarantee a certain degree of objectivity. Table 3 indicates that the anti-correlation of $\mathrm{M} \geqslant 8.2$ earthquakes with syzygies shown in Table 2 extends all the way back to 1835 , reaching its peak in 1905-1918 when the number of earthquakes in $\mathcal{H}_{3}$ was merely 3 out of 7 , or $42.9 \%$ much less than predicted by (1). The 1919-1933 years seem to be the transition period between the 1898-1918 core of anti-correlation and the correlation season of 1938-present. The $1835-1897$ years seem to be the transition period between the 1898-1918 core of anti-correlation and the correlation season of 1550 1834.

Table 4, showing $M \geqslant 8.2$ earthquakes from NOAA and GHEA, allows us to zoom in on the 1550-1833 years. The New Madrid earthquakes have been since downgraded to lower magnitudes and the $1761 / 3 / 30,1716 / 2 / 11,1586 / 7 / 10,1584 /$ $3 / 17$ earthquakes seem to be aftershocks of preceding earthquakes; hence they are moved to the bottom of the table. The $1818 / 11 / 8,1793 / 2 / 17$, $1787 / 3 / 28,1725 / 2 / 1,1647 / 5 / 14$ earthquakes $\operatorname{did}$ not make it to $\mathcal{H}_{3}$ but were close. Over all, Table 4 confirms the correlation of $\mathrm{M} \geqslant 8.2$ earthquakes with syzygies in 1550-1761. The 1793/2/17, 1792/8/22, 1784/5/13 earthquakes cluster together; they struck in or right after the 1781-1789 season of intensive geomagnetic storms and may have been caused by them, [Vaquero and Trigo, 2005]; it is also possible the beginning of the transition period of 1835-1897 should be extended from 1835 to $1755-1762$. Of the 24 earthquakes in 1555 $1755,22 / 1815 / 12$, or $91.7 \% / 75 \% / 63 \% / 50 \%$ are in $\mathcal{H}_{3} / \mathcal{H}_{2} / \mathcal{H}_{1} / \mathcal{H}_{0}$, the ratios of these percentages to (1) $\kappa_{3}=\frac{55.6}{74.1} \approx 1.24, \kappa_{2}=\frac{44.4}{60.6} \approx 1.24, \kappa_{1}=$ $\frac{29.6}{47.1} \approx 1.34, \kappa_{0}=\frac{22.2}{33.7} \approx 1.48$ increase as $\nu$ deceases. The 1755-1762 years separating the 1555 1755 and $1762-1835$ periods are chronologically close to $1753-1755$ when the magnetic poles' paths changed their directions as shown in Figure 1.

\section{The year 2012 phenomenon}

A strong connection to syzygy-closest perigees was exhibited by the strongest earthquakes of the full lunar cycle in 2009/7/21-2018/7/13 when the strongest earthquake of the full lunar cycle struck within 33 days of Full Moon-closest perigee in six out of eight full lunar cycles, as shown in Table 5. The correlation is, most likely, due to Full Moon coming within less than 66 minutes of the closest perigee of the full lunar cycle for five years in a row: on $2011 / 3 / 19,2012 / 5 / 6,2013 / 6 / 23$, 2014/8/10, 2015/9/28 Full Moon and the closest perigee were correspondingly $59,2,23,27$, and 65 minutes apart; such an event is very rare and might be the reason why the ancient Maya used 2012 as a time stamp to mark the end of one time cycle and the beginning of another, hence we refer to the event as the year 2012 phenomenon. The previous sequence of Full Moon-closest/2nd closest perigees of less than an hour between Full Moon and perigee was on $1809 / 5 / 29,1810 / 7 / 16$, $1811 / 9 / 2,1812 / 10 / 20$, followed by Full Moon-closest perigees of $356496 \mathrm{~km}$ on $1813 / 12 / 7$ and of 56647 $\mathrm{km}$ on $1815 / 1 / 25$; it was followed by the $1815 / 4 / 10$ $\mathrm{VEI}=7$ eruption of Tambora. Would there be a powerful eruption in a year or two?

The full lunar cycles started and ended on the day of New Moon-2nd closest perigee in 2009/7/21$2012 / 12 / 12$, in $2014 / 1 / 30-2018 / 8 / 10$, the full lunar cycles started and ended one synodic after New Moon-2nd closest perigee; that may have contributed to the breakdown in the pattern causing the strongest earthquake to strike on $2014 / 4 / 1$ rather than within 33 days of the 2014/8/10 Full Moon-closest perigee.

The years 2010-2014 produced five $M \geqslant 8.2$ earthquakes, averaging one per year; for comparison, in 1900-2009 there were only $39 \mathrm{M} \geqslant 8.2$ 

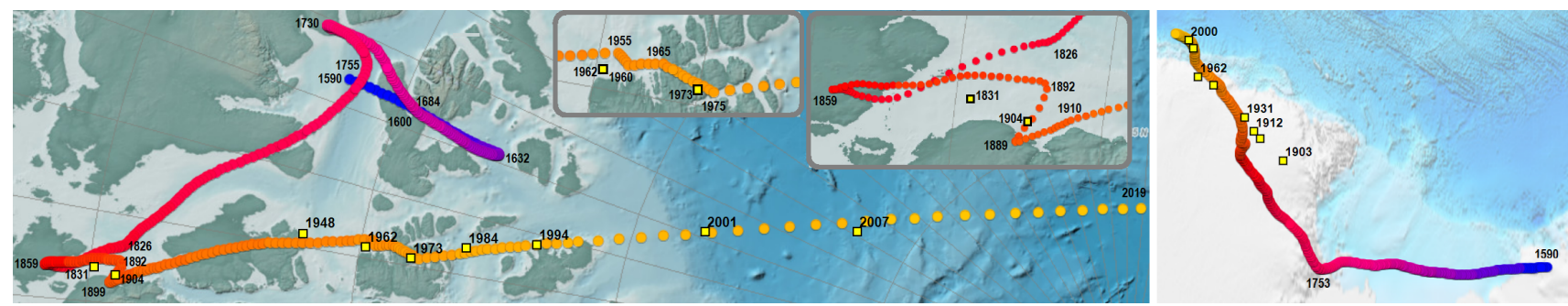

Figure 1. Modelled path of the magnetic poles; yellow squares indicate observed locations, https://maps.ngdc.noaa.gov/viewers/historical_declination/. The gUFM model was used for 1590-1890, the IGRF model was used for 1900-2020, a smooth transition was imposed for 1890-1900 to connect the models. The modelled path often significantly deviates from the observed locations, and thus should be viewed only as a rough approximation of the real path. The 1859 turn in the path coincided with the Carrington solar storm of 1859/9/1-2; the 1730 turn in the path coincided with the Boston solar storm of $1730 / 10 / 22$. The paths of both poles made significant turns in 1753-1755.

earthquakes averaging less than 0.36 earthquakes per year. Of the thirteen $\mathrm{M} \geqslant 8.6$ earthquakes in 1900-2017, three, or 23\%. struck in 2010-2012. In the $2017 / 5 / 26-2018 / 7 / 13$ full lunar cycle, the second strongest earthquake was 21 days after Full Moon-closest perigee, the strongest earthquake of the lunar cycle struck within two days of $2017 / 9 / 6$ Full Moon and 2017/9/7 X9.3 solar flare.

In the 2008/6/3-2009/7/21 full lunar cycle, the strongest earthquake was of $M=7.8$ and it struck on $2009 / 7 / 15,6$ days before the $2009 / 7 / 21$ syzygyperigee; it was preceded by a $\mathrm{M}=7.7$ earthquake on 2009/7/5, 16 days before the 2009/7/21 syzygyperigee. Another $\mathrm{M}=7.7$ earthquake struck on 2009/1/3, 22 days after the 2008/12/12 Full Moonclosest perigee. In the $2007 / 4 / 17-2008 / 6 / 3$ full lunar cycle, the strongest earthquake was of $\mathrm{M}=8.4$ and it struck on $2007 / 9 / 12,44$ days before the 2007/10/26 Full Moon-closest perigee. So the pattern was building up two full lunar cycles prior to 2009/7/21-2018/7/13. We may expect the pattern to wither after $2018 / 7 / 13$ but it still should be felt in the 2018/7/13-2019/8/30 full lunar cycle suggesting a powerful earthquake of $M \geqslant 7.7$ close to the 2019/2/19 Full Moon-closest perigee.

\section{$M \geqslant 6.6$ earthquakes in Africa}

The East African Rift Line is only a forming tectonic line, the earthquakes along it cannot be attributed to the motion of continental plates and may be expected to be more influenced by syzygies than earthquakes elsewhere. Africa's 19002017 earthquakes listed by USGS as $\mathrm{M} \geqslant 6.6$ are shown in Table 6 . Of the total of 18 earthquakes, $15 / 15 / 11 / 10$, or $83.3 \% / 83.3 \% / 61.1 \% / 55.6 \%$, were in $\mathcal{H}_{3} / \mathcal{H}_{2} / \mathcal{H}_{1} \mathcal{H}_{0}$, the ratios of these percentages to $(1) \kappa_{3}=\frac{83.3}{74.1} \approx 1.12, \kappa_{2}=\frac{83.3}{60.6} \approx 1.37, \kappa_{1}=$ $\frac{61.1}{47.1} \approx 1.3, \kappa_{0}=\frac{55.6}{33.7} \approx 1.65$ may be said to "almost increase" as $\nu$ decreases. Of the ten earthquakes along the East African Rift Line, shown in Table 6 in bold, $8 / 8 / 6 / 6$, or $80 \% / 80 \% / 60 \% / 60 \%$, were in $\mathcal{H}_{3} / \mathcal{H}_{2} / \mathcal{H}_{1} \mathcal{H}_{0}$; the ratios of these percentages to $(1) \kappa_{3}=\frac{80 .}{74.1} \approx 1.08, \kappa_{2}=\frac{80 .}{60.6} \approx$ $1.32, \boldsymbol{\kappa}_{1}=\frac{60 .}{47.1} \approx 1.27, \kappa_{0}=\frac{60}{33.7} \approx 1.78$ may be said to "almost increase" as $\nu$ decreases. The 1990/7/9 earthquake was 107 minutes short of $\mathcal{H}_{1}$, had it struck 107 minutes earlier, both ratios would have been increasing as $\nu$ decreases. Three more earthquakes listed as $\mathrm{M} \geqslant 6.6$ by NOAA are shown at the bottom of Table 6 .

\section{Powerful earthquakes at great depths}

Most earthquakes strike in or near the crust and/or upper mantle with the focal depth in the range of $0-400 \mathrm{~km}$; yet some have focal depth up to $700 \mathrm{~km}$, striking in the transition zone separating the upper mantle from the lower mantle. The high temperature and pressure in the transition zone should make them more susceptible to tidal forces.

Table 7 shows $\mathrm{M} \geqslant 7.4$ earthquakes of the focal depth $\geqslant 400 \mathrm{~km}$ according to USGS and NOAA in 1900-2018/8/1. Of the 22 earthquakes from 
USGS, $18 / 16 / 12 / 7$, or $81.8 \% / 72.7 \% / 54.5 \% / 31.8 \%$ are in $\mathcal{H}_{3} / \mathcal{H}_{2} / \mathcal{H}_{1} / \mathcal{H}_{0}$; of the 28 earthquakes from NOAA $23 / 19 / 14 / 9$, or $82.1 \% / 67.9 \% / 50 \% / 32.1 \%$, are in $\mathcal{H}_{3} / \mathcal{H}_{2} / \mathcal{H}_{1} / \mathcal{H}_{0}$; both distributions are somewhat similar to (1). A bit more careful examination of Table 7 shows that all earthquakes that did not make it to $\mathcal{H}_{3}$, almost did so. Three earthquakes $1963 / 8 / 15,1958 / 7 / 26,1957 / 9 / 28$ that $\operatorname{did}$ not make it to $\mathcal{H}_{3}$ were at the time of the most powerful primary solar cycle.

\section{VEI $\geqslant 5$ volcanic eruptions in $1600-2017$}

Unlike earthquakes, most powerful volcanic eruption leave long-lasting traces letting us determine their VEI and dates. Although the dates and VEI of powerful eruptions can be determined sufficiently well, it is hard to determine when the seismic activity associated with such eruptions actually started as powerful eruptions are often preceded by earthquakes and less powerful eruptions; with that in mind, Table 8 shows all VEI $\geqslant 5$ eruptions in 1600-2017, with the date and time selected or estimated to be those of the strongest blast, unless otherwise stated.

The number of eruptions in $\mathcal{H}_{3} / \mathcal{H}_{2} / \mathcal{H}_{1} / \mathcal{H}_{0}$ in $1600-1815$ was $14 / 12 / 10 / 8$ or $82.4 \% / 70.6 \% / 58.8 \% /$ $47.1 \%$ of the total of 17 ; the ratios of these percentages to $(1) \kappa_{3}=\frac{82.4}{74.1} \approx 1.11, \kappa_{2}=\frac{70.6}{60.6} \approx$ $1.165, \kappa_{1}=\frac{58.8}{47.1} \approx 1.25, \kappa_{0}=\frac{47.1}{33.7} \approx 1.4$ increase as $\nu$ decreases.

The number of eruptions in $\mathcal{H}_{3} / \mathcal{H}_{2} / \mathcal{H}_{1} / \mathcal{H}_{0}$ in 1913-2017 was $8 / 7 / 5 / 4$ or $72.7 \% / 63.6 \% / 45.5 \% /$ $36.4 \%$ of the total of 11 ; which is almost identical to (1) and the the ratios of these percentages to $(1) \kappa_{3}=\frac{72.7}{74.1} \approx 0.98, \kappa_{2}=\frac{63.6}{60.6} \approx 1.05, \kappa_{1}=$ $\frac{45.5}{47.1} \approx 0.97, \kappa_{0}=\frac{36.4}{33.7} \approx 1.08$ are all close to one. Yet the three eruptions that did not make it to $\mathcal{H}_{3}$ were close, so $\kappa_{3} \approx \kappa_{2} \approx \kappa_{1} \approx 1$ might be due to lack of refinement of the method employed in this paper rather than due to lack of correlation. The $1963 / 3 / 17$ eruption of Agung occurred right in the midst of the $1957 / 3 / 9-1965 / 1 / 2 / 24$ period which had nine $\mathrm{M} \geqslant 8.2$ earthquakes, most likely caused by the 19th primary solar cycle, the most powerful primary solar cycle known since the record-keeping started in 1700 .
Table 9 shows that each VEI $\geqslant 6$ eruption was preceded by additional celestial events which either increased the tidal pull, or applied the tidal pull periodically several times, or added electromagnetic force to the tidal force; these events are not taken into account by $\mathcal{H}_{\nu}$ 's.

During the anti-correlation season of 1822-1912, 8 out of 9 volcanic eruptions, or $88.9 \%$ were outside of $\mathcal{H}_{3}$; that is $\frac{88.9}{100-74.1} \approx 3.4$ times more than what a random distribution of eruptions would have produced.

All but one known $\mathrm{M} \geqslant 8.2$ earthquakes in 1687 1755 and all but two known VEI $\geqslant 5$ volcanic eruptions in 1707-1815 occurred within 30 days of a syzygy-closest/2nd closest perigee; all of them occurred within 3.5 days of a regular syzygy or within 33.5 days of a syzygy-perigee.

\section{Antipodal symmetry of earthquakes and eruptions}

Since the tidal forces produced by the Moon, and amplified near syzygies, are almost antipodally symmetric, we may expect the regions of powerful seismic activity to show antipodal symmetry, i.e. the most powerful seismic activity may be expected to be accompanied by considerable seismic activity near the antipodal location. Table 10 shows that this is indeed the case. Not only the $\mathrm{M} \geqslant 8.6$ earthquakes correlate with syzygies but so do their almost antipodal matches.

Magnitude $\geqslant 8.2$ earthquakes in 1900-2017, according to USGS, are shown in Figure 2 by circles. They may be divided into groups based on location: 1) in or close to South America or its antipode, marked purple; 2) over or near the shallow floor of the Scotia Sea, shown in an inset, or its antipode, marked brown; 3) over or close to the shallow floor of Zealandia, shown in an inset, or its antipode, marked green; 4) along the Kuril-Japan and Aleutian trenches or close to Alaska, marked yellow.

NOAA Catalogue assigns magnitude $\geqslant 8.2$ to additional earthquakes: 1969/8/11, 1959/5/4, $1948 / 1 / 24, \quad 1943 / 4 / 6, \quad 1942 / 8 / 24,1941 / 11 / 25$, $1939 / 1 / 25, \quad 1924 / 4 / 14, \quad 1919 / 1 / 1, \quad 1918 / 9 / 7$, $1917 / 6 / 26,1908 / 12 / 12,1907 / 4 / 15,1906 / 12 / 12$, $1906 / 1 / 21, \quad 1905 / 1 / 22,1904 / 8 / 27,1904 / 6 / 25$, 


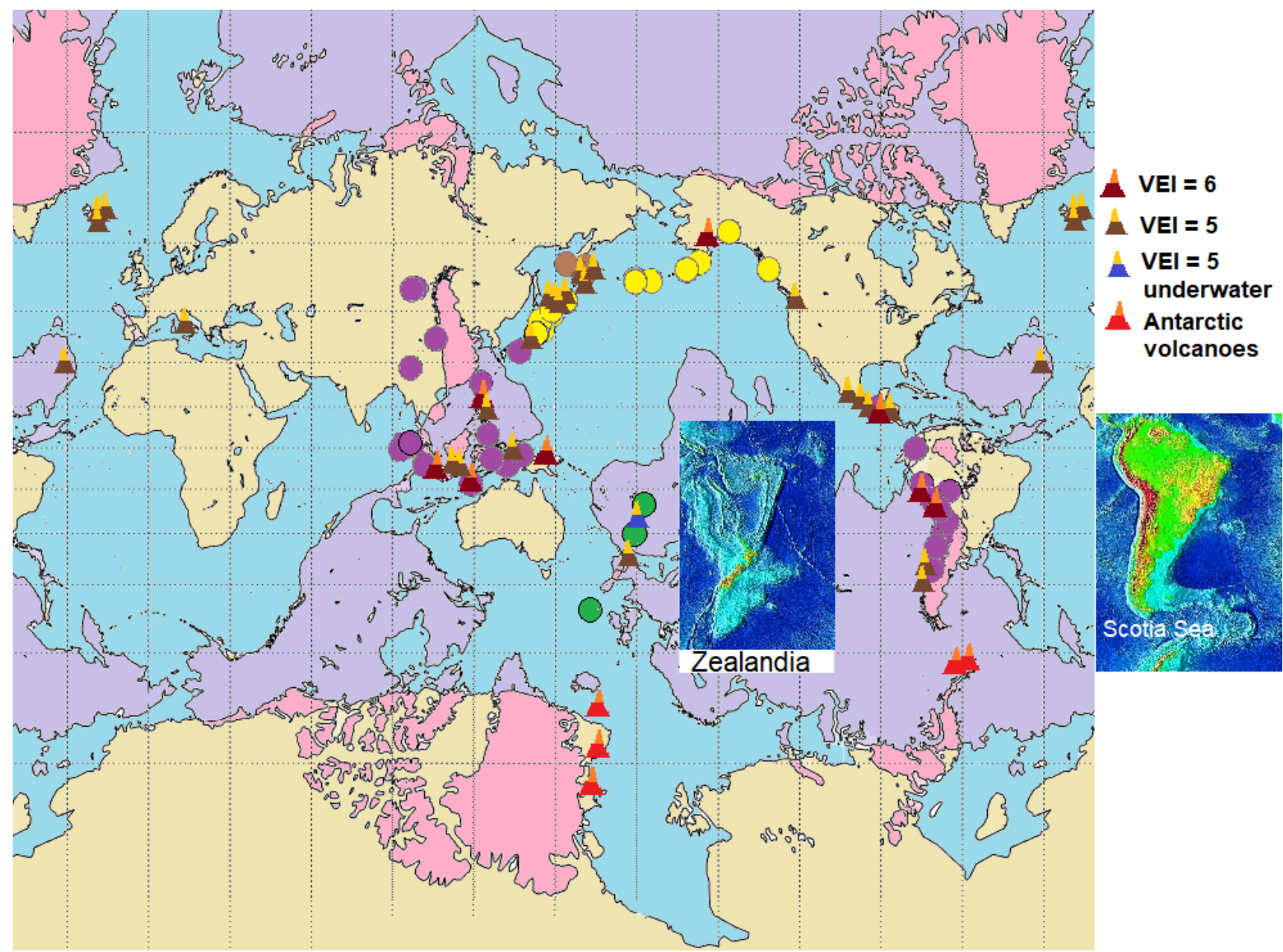

Figure 2. Seismic activity on the map of antipodes in Mercator projection. The land antipodal to water is shown in light sandy color, water antipodal to land is shown in light purple, land antipodal to land is shown in pink, water antipodal to water is shown in blue. Magnitude $\geqslant 8.2$ earthquakes in 1900-2017, according to, are shown by circles. Known VEI $\geqslant 5$ eruptions and five known Antarctic eruptions in 1892-2018 are shown by the symbols of volcanoes.

1903/6/2, 1901/8/9 in Japan, 1901/8/9 Loyalty Island, $1900 / 10 / 29$ and $1900 / 10 / 9$; each one of them fits into either of the categories marked purple, green, brown or yellow.

VEI $\geqslant 5$ volcanic eruptions in 1600-2017 are shown in Figure 2 by symbols of volcanoes. The majority struck on or near land or shallow water antipodal to land or shallow water or along the Kuril-Japan and Aleutian trenches or in Alaska; the only exception is the 1980/5/18 eruption of St. Helens. The only five surface Antarctic volcanoes, known to erupt in 1892-2018, are near the land antipodal to land.

Most of powerful seismic activity occurs in or close to South and Central America or its antipodes and along the Kuril-Japan and Aleutian trenches and close to Alaska. The earthquakes in continental Asia and the volcanic eruptions in Iceland, Italy and the Atlantic are not in the Pacific Ring of Fire but their antipodes are.

\section{The correlation pattern and the Earth's magnetic field}

The anti-correlation of earthquakes to syzygies in 1835-1933 in Table 1, Table 2, Table 3, and anti-correlation of volcanic eruptions to syzygies in 1822-1912 in Table 8 are chronologically close to the chaotic part the magnetic North pole's path in 1826-1910 seen in Figure 1; the three intervals are also close to a secondary solar cycle $\approx 1800-1910$ 


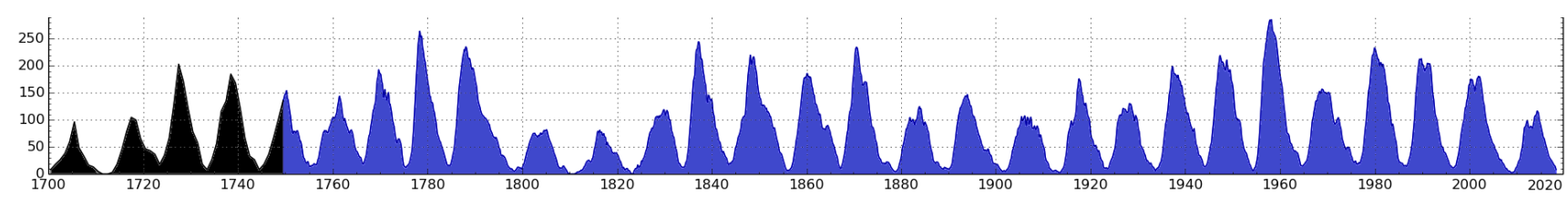

Figure 3. Yearly mean sunspot number (black) up to 1749 and monthly 13month smoothed sunspot number (blue) in 1749-2017, http://www.sidc.be/silso/ yearlyssnplot. The maxima of the primary solar cycles rise and fall in somewhat cyclical manner known as the secondary solar cycles.

in Figure 3, the longer period 1784-1933 of anticorrelation of earthquakes to syzygies suggested by Table 4 is also close to the secondary solar cycle 1800-1930. That suggests a connection between secondary solar cycles and seismic activity, but with so little data available any attempts to draw a conclusion would be premature.

Several more aspects of the magnetic North Pole seem to mirror seismic activity: 1) the $1647 / 5 / 14$ earthquake and 1631/12/16 eruption occurred close to the 1632 sharp turn in the path of the magnetic North pole at the beginning of the Maunder minimum; 2) the 1977/8/19 earthquake in Table 1 and the worsening of correlation of volcanic eruptions to syzygies in 1956-1980 in Table 8 were chronologically close to the change in the direction of motion of the magnetic North pole around 1955-1975 shown in the inset; 3 ) the 1684-1755 angle in the path of the North magnetic pole coincides with a period of extremely good correlation in Table 3 and Table 8.

Figure 4 shows $M \geqslant 8.2$ earthquakes and VEI $\geqslant 5$ volcanic eruptions in 1958-2016 versus cosmic ray intensity (CRI). All the eruptions and most of the earthquakes occurred close to drop-downs in $\mathrm{CRI}$; one may be tempted to conclude that the
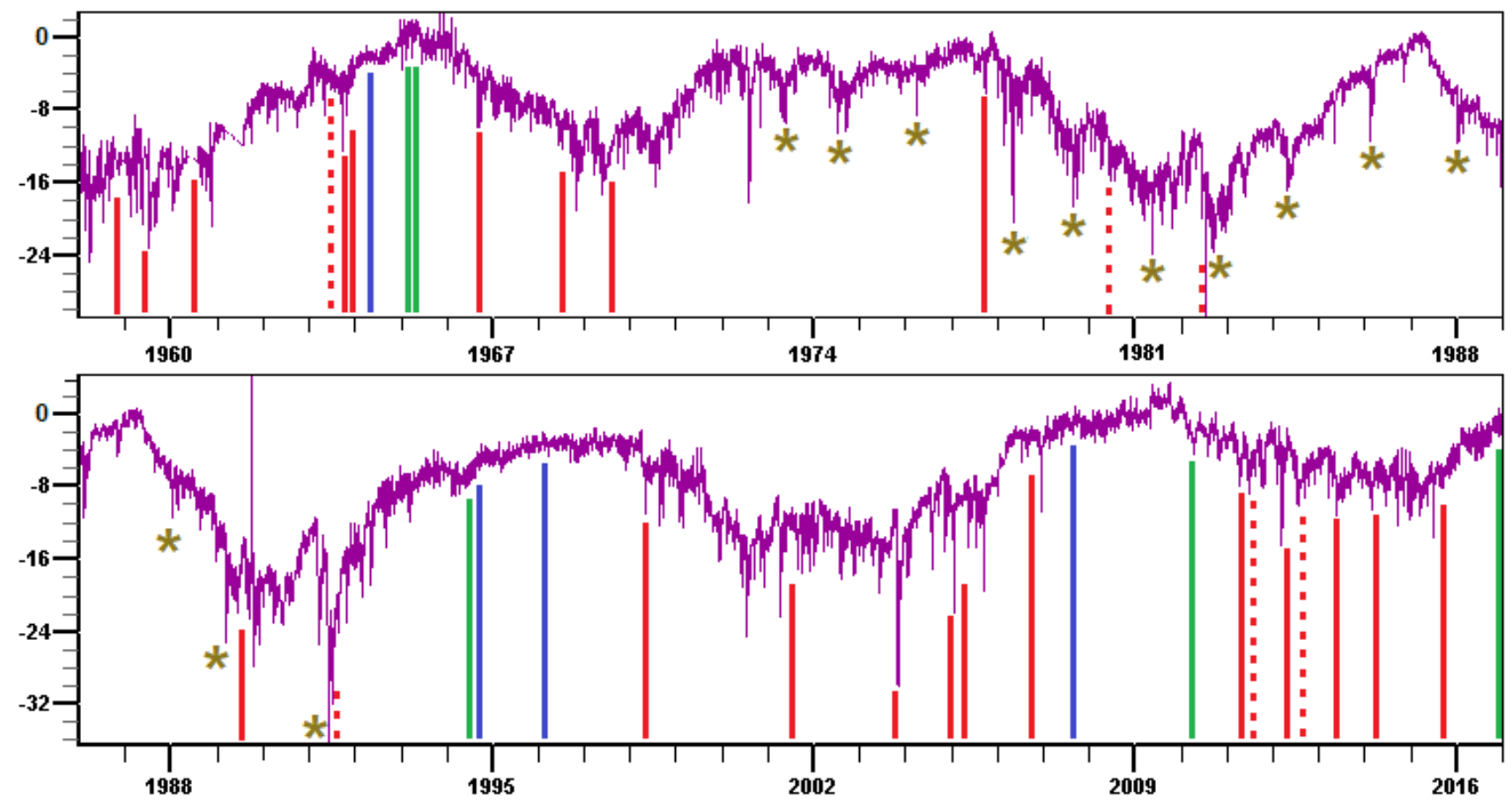

Figure 4. $M \geqslant 8.2$ earthquakes and $V E I \geqslant 5$ volcanic eruptions versus cosmic ray intensity (CRI) in 1958-2016. Daily average of CRI is shown in purple. M $\geqslant 8.2$ earthquakes are marked by solid vertical lines, red lines indicate earthquakes near considerable drop-downs in CRI, green lines indicate earthquakes near insignificant drop-down, blue lines indicate earthquakes far away from any drop-downs. VEI $\geqslant 5$ volcanic eruptions are marked by dotted vertical lines, all of them are near drop-downs in CRI. The recurring drop-downs in 1973/5/17-1991/6/15 are marked by asterisks. (After http://cr0.izmiran.ru/mosc/ ) 
drop-downs contributed to the powerful seismic events. However, the most pronounced drop-downs in CRI recurred in 1973/5/17-1991/6/15 approximately every 600 days, yet only two $\mathrm{M} \geqslant 8.2$ earthquakes and only two VEI $\geqslant 5$ volcanic eruptions occurred at that time. The drop-downs in CRI are usually caused by solar flares, yet some drop-downs follow earthquakes rather than precede them suggesting that there might be a third agent affecting both the earthquakes and the solar activity leading to the solar flares. That certain terrestrial activities seem to precede solar activities was also pointed out in [Hathaway and Wilson, 2006.

Drop-downs in CRI may also be due to changes in the geomagnetic field. The existence of correlation between powerful seismic activity and drastic changes in the geomagnetic field is supported by other observations, e.g. 1) the eruption of Pinatubo, the most powerful eruption of the past 60 years coincided with a drastic increase in solar flare, [Kovalyov, 2019, Table 8], which certainly affected the Earth's magnetic field; 2) 2004/12/27 powerful $\gamma$-ray burst practically coincided with the 2004/12/26 $\mathrm{M}=9.1$ earthquake; 3) powerful solar flares in March-April of 1950 preceded the $1950 / 5 / 22 \mathrm{M}=9.5$ earthquake; 4) the only two earthquakes in Table 5 that did not strike within 31 days of perigee-syzygy, struck 1-3 days after considerable solar flares.

Is there a deep-rooted relationship between the seismic and geomagnetic activities? The geophysicists of today do not believe so and attribute any correlation between the geomagnetic and seismic activities to a mere coincidence. But if the most powerful seismic events draw their power from the liquid core, then the seismic activity should be affected by both the tidal and electromagnetic forces for the liquid core is affected by both.

\section{Discussion}

The weather forecast involves work with numerous factors affecting the weather, the seismic forecast should also be based on numerous factors. Yet a number of researchers persistently fixate on syzygies alone in their attempts to show lack of correlation of seismic activity with syzygies. Different aspects of earthquakes depend on whether the earthquakes are intraplate or interplate, shallow or deep, in ocean or on land; they are affected by cos- mic rays, solar flares and coronal mass ejections, as well as lunar syzygies. Sometimes the influence of syzygies becomes dominant making correlation of seismic activity with syzygies more clear.

The syzygies themselves are not the same and affect seismic activity differently, depending on the distance from Earth, time to the nearest perigee, the subsolar and sublunar points, and much more. A very rudimentary and simplistic attempt to account for these differences is the separation of syzygies into regular syzygies and syzygy-closest/2nd closest perigees employed in this paper. Failure to differentiate between regular syzygies and syzygyclosest/2nd closest perigees leads to the seismic events within $30+\nu$ days of syzygy-closest/2nd closest perigees, spread out more or less uniformly over different lunar phases, overshadowing any correlation between regular syzygies and seismic events. The tools used in this paper might be the first, albeit rudimentary, tools to study the effect of tidal forces on seismic activity. To develop better tools, one needs to study patterns of seismicity; yet with only 50 years of totally reliable seismic data and another 50 years of relatively reliable seismic data available, only few patterns of seismic activity are easily detectable.

The seismic activity appears to be sensitive to both the tidal forces exhorted by Moon and Sun and the electromagnetic forces exhorted by solar flares and cosmic rays. There is only one part of Earth sensitive to both, and that is the liquid core, suggesting that, at least some, powerful earthquakes draw their power from the liquid core. Recent work [Gardonio et al., 2018] suggests that, at least some, earthquakes may be caused by pulses of deep fluids. We believe the fluid is coming from the liquid core.

Acknowledgments. The author would like to thank Selena and Nickolas Kovalyov who helped with the preparation and verification of the tables and data analysis. The author would like to thank the organizations and individuals who produced the data and software used in the paper. The author would also like to thank the reviewers and editors for helpful suggestions. Especial thanks go to Dr. Vitaly Nechitailenko who thoroughly checked and corrected the manuscript and formatted the manuscript and patiently guided the author through the preparation of the manuscript for publication. 


\section{References}

Gardonio, B., R. Jolivet, E. Calais, H. Leclere (2018), The April $2017 \mathrm{M}_{w} 6.5$ Botswana Earthquake: An Intraplate Event Triggered by Deep Fluids, Geophysical Research Letters, 45, No. 17, 8886-8896, Crossref

Hathaway, D., R. Wilson (2006), Geomagnetic activity indicates large amplitude for sunspot cycle 24, Geophysical Research Letters, 33, No. 18, Crossref

Klotz, O. (1914),Earthquakes, phases of the moon, sublunar and sub-solar points, Journal of the Royal Astronomical Society of Canada, 8, 273-281. (http:// articles.adsabs.harvard.edu//full/1914JRASC...8..273 K/0000273.000.html
Kovalyov, M. (2019), Seismic activity and syzygies data, Earth Science DataBase, GC RAS, Moscow. Crossref

Vaquero, J., R. Trigo (2005), Auroras Observed in Portugal in Late 18th Century, Obtained from Printed and Manuscript Meteorological Observations, Solar Physics, 231, 157-166. (The numerous lowlatitude auroras in 1781-1789 are indicative of powerful geomagnetic storms. http://idlcc.fc.ul.pt/pdf/Va quero_Trigo_2005_Solar_Physics.pdf)

\section{Corresponding author:}

Mikhail Kovalyov, Professor Emeritus, University of Alberta, Edmonton, Alberta, Canada. (mkovalyo[at] ualberta.ca) 